\title{
The Neuroimaging Signature of Frontotemporal Lobar Degeneration Associated with Granulin Mutations: An Effective Connectivity Study
}

\author{
Enrico Premi ${ }^{1}$, Mario Grassi ${ }^{2}$, Stefano Gazzina ${ }^{1}$, Barbara Paghera ${ }^{3}$, Daniele Pepe ${ }^{2}$, Silvana Archetti ${ }^{4}$, \\ Alessandro Padovani ${ }^{1}$, and Barbara Borroni ${ }^{1}$ \\ ${ }^{I}$ Centre for Ageing Brain and Neurodegenerative Disorders, Neurology Unit, University of Brescia, Italy; ${ }^{2}$ Department of \\ Epidemiology and Statistics, University of Pavia, Italy; ${ }^{3}$ Nuclear Medicine Unit, University of Brescia, Italy; and ${ }^{4}$ Department \\ of Laboratories, III Laboratory of Analysis, Brescia Hospital, Brescia, Italy
}

\begin{abstract}
It has been suggested that monogenic frontotemporal lobar degeneration (FTLD) due to Granulin (GRN) mutations might present a specific pattern of atrophy, as compared with FTLD GRN-negative disease. Recent literature has suggested that the study of functional neural networks, rather than regional structural damage, might better elucidate the pathogenic mechanisms, showing complex relationships among structural alterations observed with conventional neuroimaging. The aim of this study was to evaluate effective brain connectivity in FTLD patients carrying GRN mutations (GRN+), compared with FTLD patients without pathogenetic GRN mutations $(G R N-)$ and healthy controls (HCs). Methods: Twenty-six FTLD patients (13 GRN+ and 13 GRN - matched for age, sex, and phenotype) and 13 age- and sex-matched HCs underwent brain perfusion SPECT. Brain regions involved in FTLD (dorsolateral, anterior cingulate, orbitofrontal, posterior temporal, temporal pole, and parietal) were used as regions of interest to identify functionally interconnected areas. An effective connectivity (path) analysis was defined with a PC algorithm (named after its inventors Peter Spirtes and Clark Glymour) search procedure and structural equation fitting. Statistically significant differences among the 3 groups were determined. Results: The best-fitting model was obtained by the data-driven approach, and brain connectivity pathways resembling state-of-the-art anatomic knowledge were obtained. When $G R N+$ and GRN - groups were considered, the former presented a selective bilateral parietotemporal disconnection, compared with GRN- patients. Furthermore, in FTLD GRN+ patients an increased compensative connectivity of the temporal regions (temporal pole and posterior temporal cortices) was observed. Conclusion: The present work suggests that impairment of effective functional connectivity of the parietotemporal regions is the hallmark of $G R N$ related FTLD. However, compensative mechanisms - which should be further investigated-may occur.
\end{abstract}

Key Words: frontotemporal dementia; frontotemporal lobar degeneration; granulin; mutation; SPECT; path analysis

J Nucl Med 2013; 54:1066-1071

DOI: 10.2967/jnumed.112.111773
$\mathbf{F}$ rontotemporal lobar degeneration (FTLD) is a common cause of young onset neurodegenerative dementia $(1,2)$. FTLD is clinically and pathologically heterogeneous (3), and genetic factors play a significant role, with a genetic basis implicated in up to $40 \%$ of cases (4).

Discovery of disease-causing mutations in the Granulin (GRN) and microtubule-associated protein $\tau$ - $(M A P T)$ genes and the expanded hexanucleotide repeat insertion within $C 9$ orf 72 have allowed a giant step forward in the knowledge of causative mechanisms of FTLD. Accordingly, recent literature has carefully detailed the peculiar features of these genetic forms, with the attempt to facilitate early and accurate diagnosis.

Autosomal-dominant inherited GRN disease has been associated with a varied clinical spectrum, ranging from behavioral variant frontotemporal dementia (bvFTD) to nonfluent/aggramatic subtypes of primary progressive aphasia (nfvPPA), and with a widespread and asymmetric pattern of atrophy that involved frontal, temporal, and parietal lobes (5-8). In light of these findings, it has been suggested that imaging could be useful to help predict $G R N$ mutations and hence the underlying pathology. Thus, characterizing the patterns of atrophy associated with inherited disorders provides important insights into pathologic mechanisms. Despite the considerable neurobiologic and clinical interest in delineating the signature of structural damage associated with FTLD, the role of neuronal network breakdown has attracted much recent interest (9-11), because it might antedate regional atrophy. Statistical approaches based on structural equation modeling (SEM) might be of help in assessing the complex relationship within pathways of interest, considering single anatomic regions as a part of functional connections where specific alteration influences the entire network (12).

In the present study, we therefore used connectivity techniques to identify the critical neural links that differentiated autosomaldominant $G R N$-related disease. To capture $G R N$-associated changes, we applied voxelwise covariance analysis and SEM to SPECT data.

\section{MATERIALS AND METHODS \\ Subjects \\ We identified all patients seen at the Centre for Ageing Brain and Neurodegenerative Disorders, University of Brescia, Italy, who had been screened for mutations in GRN with a SPECT imaging scan. All}


TABLE 1

Demographic and Clinical Characteristics of Included Subjects

\begin{tabular}{|c|c|c|c|c|}
\hline Variable & FTLD GRN $+(n=13)$ & FTLD $G R N-(n=13)$ & $\mathrm{HC}(n=13)$ & $P$ \\
\hline Age at scan $(y)$ & $58.1 \pm 5.1$ & $60.6 \pm 5.1$ & $60.7 \pm 10.0$ & $0.86^{\star}$ \\
\hline Age at onset $(y)$ & $56.1 \pm 5.8$ & $58.5 \pm 4.9$ & - & 0.27 \\
\hline Onset to scan $(y)$ & $2.1 \pm 2.0$ & $2.2 \pm 1.3$ & - & 0.91 \\
\hline Sex, male & $46.2 \%(6)$ & $46.2 \%(6)$ & $61.5 \%(8)$ & $0.66^{\dagger}$ \\
\hline Education (y) & $8.5 \pm 4.1$ & $6.5 \pm 2.4$ & - & 0.14 \\
\hline Family history & $84.6 \%(11)$ & $15.4 \%(2)$ & - & $0.001^{\dagger}$ \\
\hline $\begin{array}{l}\text { Frontotemporal Dementia-modified } \\
\text { Clinical Dementia Rating scale }\end{array}$ & $5.4 \pm 4.1$ & $5.6 \pm 2.9$ & - & 0.85 \\
\hline Mini-Mental State Examination & $24.1 \pm 5.1$ & $19.0 \pm 9.3$ & - & 0.10 \\
\hline Frontal Behavioral Inventory total score & $15.8 \pm 10.7$ & $14.7 \pm 9.2$ & - & 0.79 \\
\hline
\end{tabular}

*ANOVA test.

${ }^{\dagger} \chi$-square test.

Data in parentheses are no. of subjects. Results are from $t$ test, unless otherwise specified.

included patients signed a written informed consent for the present study. Thirteen FTLD patients were identified, all carrying the $G R N$ $T h r 272 f s$ mutation (FTLD GRN+). We then identified a disease comparison group of age- and sex-matched FTLD patients. These patients were negative for genetic mutations within GRN (FTLD GRN-). Furthermore, the FTLD GRN- group did not carry mutations within $M A P T$ and $C 9$ orf 72 .

$G R N+$ had either bvFTD $(n=5)$ or nfvPPA $(n=8)(13,14)$, and FTLD GRN- were matched for diagnosis as well (5 bvFTD and 8 nfvPPA).

Moreover, a group of 13 age- and sex-matched healthy controls (HCs) were included; a brief neuropsychologic assessment was administered, and HCs performed within reference ranges in all cognitive domains.

The following additional stringent exclusion criteria were applied: cerebrovascular disorders, previous stroke, hydrocephalus, and intracranial mass documented by MR imaging; a history of traumatic brain injury or another neurologic disease; significant medical problems; and major depressive disorder, bipolar disorder, schizophrenia, substance abuse disorder, or mental retardation according to criteria of the DSM-IV (15).

The work conformed to the Helsinki Declaration and was approved by local Ethical Committee of Brescia Hospital, Italy.

\section{9mTc-Ethylcysteinate Dimer SPECT Acquisition and Image Preprocessing Analysis}

Subjects received an intravenous injection of ${ }^{99 \mathrm{~m}} \mathrm{Tc}$-ethylcysteinate dimer (1,110 MBq; Neurolite [Bristol-Myers Squibb Pharma]) while they rested supine with their eyes closed in a quiet, dimly lit room. All individuals were imaged using a dual-head rotating $\gamma$-camera (VG Millenium; GE Healthcare) fitted with a low-energy, high-resolution collimator, $30 \mathrm{~min}$ after intravenous injection of ${ }^{99 \mathrm{~m}} \mathrm{Tc}$-ethylcysteinate dimer. A $128 \times 128$ pixel matrix was used for image acquisition with 120 views over a $360^{\circ}$ orbit (in $3^{\circ}$ steps) and a pixel size and slice thickness of $1 \mathrm{~mm}$, in $27 \mathrm{~min}$ or more if total counts were lower than $5 \times 10^{6}$. Images were reconstructed using a ramp filtered-backprojection and 3-dimensionally smoothed with a Metz filter (order, 3; enhancement, 1.24; full width at half maximum, $6.7 \mathrm{~mm}$; cutoff, 0.61 cycles $\mathrm{cm}^{-1}$ ). The reconstructed images were corrected for $\gamma$-ray attenuation using the Chang method (attenuation coefficient, $0.11 \mathrm{~cm}^{-1}$ ).

Statistical parametric mapping (SPM8; Welcome Department of Cognitive Neurology, University College, London) and Matlab 7.6 (MathWorks Inc.) were used for image preprocessing. Images were spatially normalized to a reference stereotactic template (Montreal
Neurologic Institute) and smoothed by a gaussian kernel of $8 \times 8 \times 8$ $\mathrm{mm}$ in full width at half maximum.

To evaluate patterns of hypoperfusion, exploratory comparisons across the different groups were made using $t$-statistics with appropriate linear contrasts. We considered any cluster above a statistical threshold set at $P$ less than 0.001 uncorrected.

We generated a region of interest (ROI) for each region involved in the FTLD pathologic process (as demonstrated by neuroimaging and neuropathologic studies in FTLD) (16-18), using WFU PickAtlas (http://www.fmri.wfubmc.edu) (19). On both sides, ROIs of the
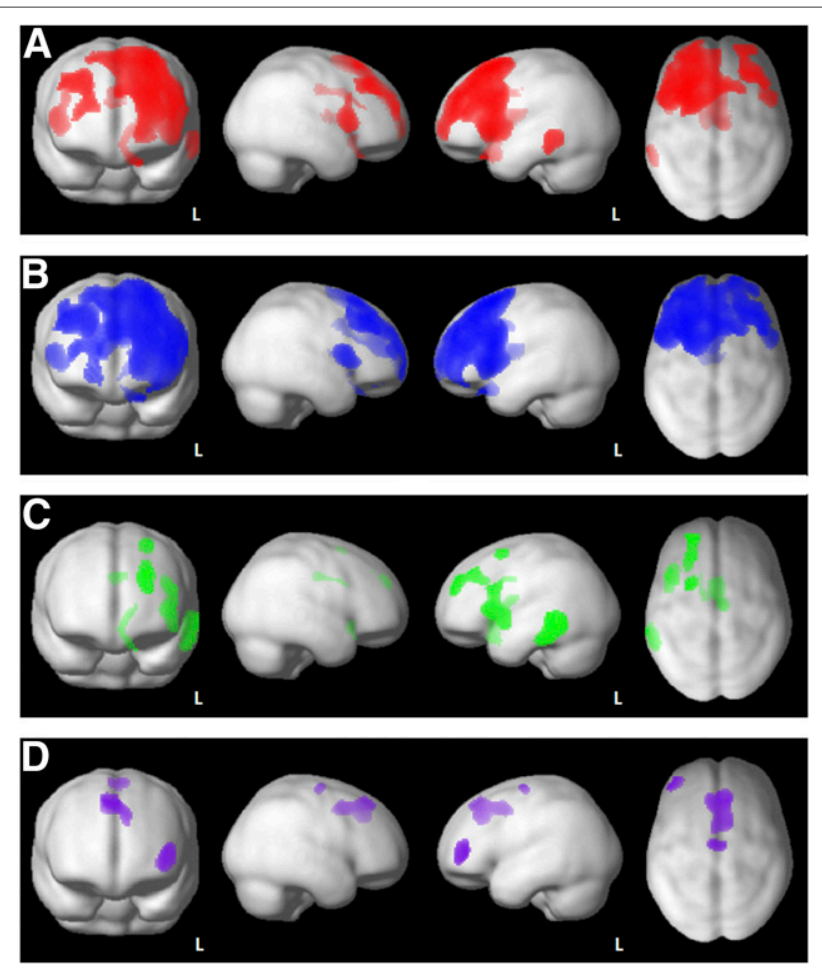

FIGURE 1. Reduction in regional cerebral perfusion in FTLD patients. Superimposed to 3-dimensional brain templates. (A) FTLD patients $(G R N+$ and $G R N-)<$ HC. (B) GRN $+<$ HC. (C) GRN $-<$ HC. (D) GRN $+<G R N-$. Threshold was set at $P<0.005$, uncorrected. 
TABLE 2

Mean Scores of ROls in 3 Groups

\begin{tabular}{|c|c|c|c|c|c|}
\hline \multirow[b]{2}{*}{ Variable } & \multirow[b]{2}{*}{ FLTD GRN+ } & \multirow[b]{2}{*}{ FLTD GRN- } & \multirow[b]{2}{*}{$\mathrm{HC}$} & \multicolumn{2}{|r|}{$P$} \\
\hline & & & & $\begin{array}{l}\text { 1-Way } \\
\text { ANOVA }\end{array}$ & $\begin{array}{l}\text { 1-Way ANOVA after correction } \\
\text { for multiple comparisons }{ }^{\star}\end{array}$ \\
\hline \multicolumn{6}{|l|}{ DLPFC } \\
\hline L & $0.83 \pm 0.09$ & $0.92 \pm 0.09$ & $0.99 \pm 0.05$ & $<0.0001$ & 0.015 \\
\hline $\mathrm{R}$ & $0.87 \pm 0.11$ & $0.96 \pm 0.09$ & $1.02 \pm 0.06$ & $<0.0001$ & 0.024 \\
\hline \multicolumn{6}{|l|}{ AntCing } \\
\hline $\mathrm{L}$ & $0.84 \pm 0.10$ & $0.92 \pm 0.13$ & $1.02 \pm 0.07$ & 0.001 & Not significant \\
\hline $\mathrm{R}$ & $0.87 \pm 0.09$ & $0.94 \pm 0.12$ & $1.02 \pm 0.08$ & 0.001 & Not significant \\
\hline \multicolumn{6}{|l|}{ Orbit } \\
\hline L & $0.88 \pm 0.10$ & $0.96 \pm 0.13$ & $1.03 \pm 0.04$ & 0.001 & Not significant \\
\hline $\mathrm{R}$ & $0.88 \pm 0.09$ & $0.95 \pm 0.11$ & $1.02 \pm 0.06$ & 0.003 & Not significant \\
\hline \multicolumn{6}{|c|}{ Temporal lobe } \\
\hline L & $0.99 \pm 0.07$ & $0.97 \pm 0.09$ & $1.06 \pm 0.04$ & 0.007 & Not significant \\
\hline $\mathrm{R}$ & $0.99 \pm 0.08$ & $1.00 \pm 0.07$ & $1.06 \pm 0.05$ & 0.016 & Not significant \\
\hline \multicolumn{6}{|l|}{ TPol } \\
\hline L & $0.80 \pm 0.06$ & $0.81 \pm 0.11$ & $0.89 \pm 0.04$ & 0.007 & Not significant \\
\hline $\mathrm{R}$ & $0.83 \pm 0.06$ & $0.85 \pm 0.10$ & $0.92 \pm 0.03$ & 0.008 & Not significant \\
\hline \multicolumn{6}{|l|}{ Parietal lobe } \\
\hline $\mathrm{L}$ & $1.02 \pm 0.08$ & $1.06 \pm 0.09$ & $1.09 \pm 0.04$ & Not significant & Not significant \\
\hline $\mathrm{R}$ & $1.03 \pm 0.08$ & $1.07 \pm 0.08$ & $1.10 \pm 0.05$ & Not significant & Not significant \\
\hline
\end{tabular}

*Bonferroni post hoc analysis between GRN+ and GRN-.

dorsolateral prefrontal cortex (DLPFC), anterior cingulate cortex (AntCing), orbitofrontal cortex (Orbit), temporal cortex (Temp), temporal pole region (TPol), and parietal cortex (Pariet) were considered. To avoid methodologic biases, the mean perfusion in the cerebellar hemispheres (left and right) was compared, and no significant differences were found. Thus, we corrected each generated ROI for cerebellar perfusion, to ensure homogeneity of the data.

\section{Path Analysis Modeling}

Path analysis was performed within the theoretic framework of SEM-a multivariate regression technique that models the covariance structure of a set of variables-and is based on a subset of possible paths connecting those variables. Unlike simple-seed ROI-based covariance analysis (functional connectivity), SEM incorporates the directional information. Path coefficients (or path weights, ranging from 0 to 1 ) represent the expected change in the activity of 1 region, given a unit change in the region influencing it and assuming that no other influencing regions had any change. Because paths reflect a direct influence of one region to another, based on total regional metabolic activity, negative path coefficients indicate ensemble inhibition and positive paths measure net excitation (12).

Effective connectivity analysis involved the following 2 major steps. First, we tested hypothesis-driven models (i.e., H models) of the connections between ROIs, based on covariance analysis and neuroanatomy literature (20-22), and then data-driven models (i.e., PC models), based on PCALG (PC algorithm) search procedures (23). The PC algorithm (named after its inventors Peter Spirtes and Clark Glymour) estimates a completed partially directed acyclic graph, using the ROI correlation matrix as input. A completed partially directed acyclic graph contains the equivalence class of undirected and directed edges (connections) between nodes (ROIs). Thus, we identified which of the candidate models (either H or PC) had the best fitting,

TABLE 3

Hypothesis Driven ( $\mathrm{HO}-\mathrm{H} 3)$ and Data-Driven (PCO-PC3) Models Fitted to Neuroimaging Data

\begin{tabular}{lccccccc}
\hline Model & Log-L & $t$ & $\begin{array}{c}\text { Akaike Information } \\
\text { Criterion }\end{array}$ & $\begin{array}{c}\text { Bayesian } \\
\text { Information Criterion }\end{array}$ & $\begin{array}{c}\text { Goodness-of- } \\
\text { fit index }\end{array}$ & $\begin{array}{c}\text { Comparative- } \\
\text { fit index }\end{array}$ & $\begin{array}{c}\text { Standardized root } \\
\text { mean square residual }\end{array}$ \\
\hline Null & -644.188 & 24 & & & & & \\
H0 & -396.537 & 29 & 851.1 & 899.3 & 0.368 & 0.609 & 0.442 \\
H1 & -327.014 & 42 & 738.0 & 807.9 & 0.472 & 0.754 & 0.149 \\
H2 & -256.931 & 46 & 605.9 & 682.4 & 0.657 & 0.911 & 0.071 \\
H3 & -252.687 & 47 & 599.4 & 677.6 & 0.674 & 0.919 & 0.058 \\
PC0 & -316.699 & 39 & 711.4 & 776.3 & 0.492 & 0.781 & 0.143 \\
PC1 & -298.970 & 40 & 677.9 & 744.5 & 0.532 & 0.821 & 0.180 \\
PC2 & -281.594 & 45 & 653.2 & 728.0 & 0.577 & 0.855 & 0.125 \\
PC3 & -250.137 & 47 & 594.3 & 672.5 & 0.684 & 0.925 & 0.052 \\
\hline
\end{tabular}

Log- $\mathrm{L}=$ loglikelihood; $t=$ no. of parameters; Null $=$ null model with zero covariances. 
according to SEM goodness-of-fit criteria (the minimum Akaike and Bayesian information scores [Akaike Information Criterion and Bayesian Information Criterion], fit indices [goodness-of-fit index and comparative fit index] $>0.90$, and standardized residual mean square residual $>0.10$ ).

Subsequently, with the selected best path model, we used a multigroup SEM method to evaluate the connection differences between groups. In the null model, the estimate of the path coefficients was considered to be equal across groups; in the alternative model, the estimate of the path coefficients was allowed to differ across groups. An omnibus test (null vs. alternative) was then performed, and statistical significance was determined by comparison of $\chi^{2}$ (X2diff) values of fit at given degrees of freedom. If there were significant difference $(P<0.05)$ in the $\chi^{2}$ goodness-of-fit index between 2 models, it could be concluded that the groups differed significantly for one or more specific connections in the context of the referred model.

The path model search and path analysis (SEM) processing were done by pcalg, a package of R 2.15.0 (R Development Core Team), and Mplus 6.1 (Muthén \& Muthén Co.), respectively.

\section{RESULTS}

\section{Subjects}

Demographic and clinical characteristics of the study participants are detailed in Table 1.

Patient groups were matched for age, sex, and disease subtype. FTLD GRN+ and FTLD GRN- did not differ for any demographic variables except for family history, which was significantly higher in the FTLD GRN+ group.

As shown in Figure 1, the main effect of diagnosis ( $G R N+$ and $G R N-<$ HC, Fig. 1A) correlates with a remarkable hypoperfusion in frontotemporal areas; this pattern was also found when the 2 subgroups of patients were compared with HCs, separately (Figs. $1 \mathrm{~B}$ and 1C). Furthermore, a main effect of mutation $(G R N+<$ $G R N^{-}$) on AntCing and left DLPFC was present (Fig. 1D). For all the aforementioned analyses, no cluster survived after correction for multiple comparisons.

As reported in Table 2, the mean scores of defined ROIs were comparable between FTLD GRN+ and $G R N-$, but the former showed a reduced ROI in DLPFC, bilaterally.

\section{Model Connectivity Search}

The previously selected 6 cortical areas were included in the path model selection using the correlation matrix of the ROIs as input. Table 3 summarizes the goodness-of-fit indices of the different models, obtained by an a priori hypothesis $(\mathrm{H} 0-\mathrm{H} 4)$ and PCALG search (PC0-PC3). Model H0 or PC0 identified the core model of hypothesis-driven and data-driven structures, respectively. H1-H3 or PC1-PC3 were those models with additional connections defined by SEM modification indices (24). The PC3 model had the best scores: minimum information criteria (Akaike Information Criterion and Bayesian Information Criterion), comparative fit index of $0.992(>0.90)$, and standardized residual mean square residual of $0.52(>0.10)$. The unidirectional (bidirectional) functional connectivity of the PC3 model is shown in Figure 2. In particular, the left and right hemispheres had the same functional connectivity: AntCing was affected by Orbit (Orbit $\rightarrow$ AntCing), whereas Temp and Tpol were the initial and final activation regions for 3 cycles: Temp $\rightarrow$ Pariet $\rightarrow$ TPol, Temp $\rightarrow$ DLPFC $\rightarrow$ Orbit TPol, and Temp $\rightarrow$ Pariet $\rightarrow$ Orbit $\rightarrow$ TPol, respectively. Interhemispherically, there were feedback links for homologous brain regions; the additional connections are reported as well.

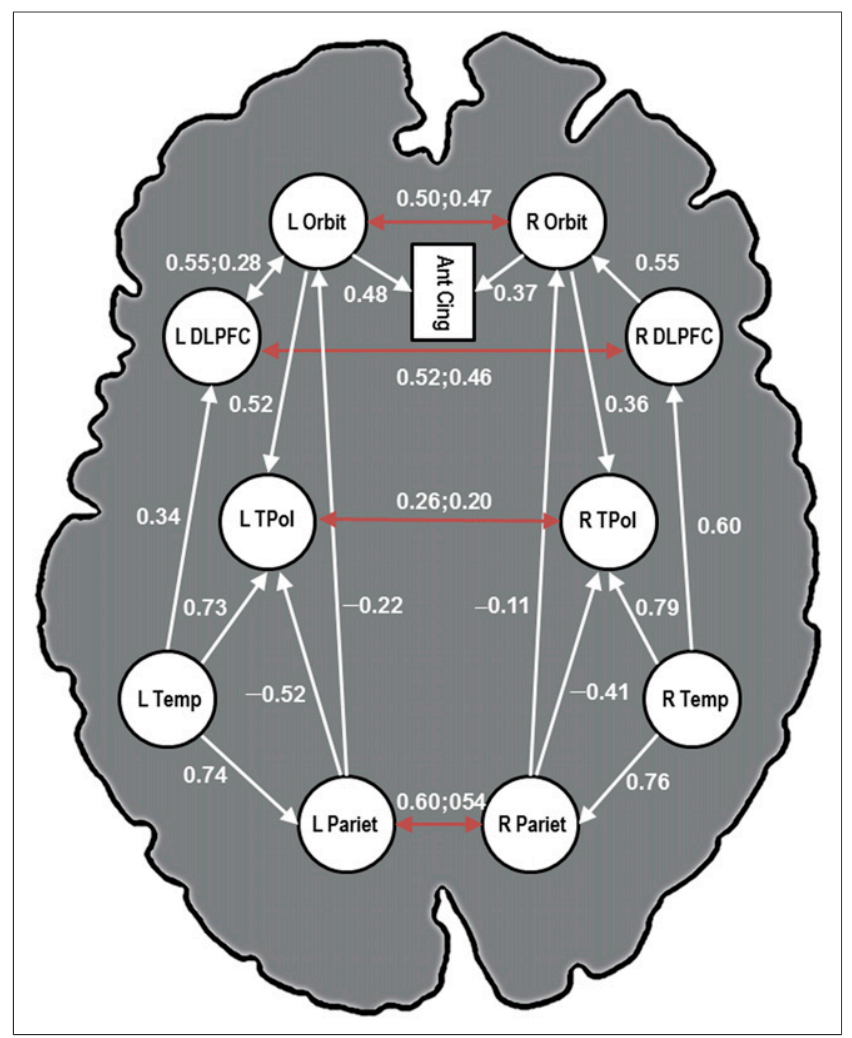

FIGURE 2. Uunidirectional (bidirectional) functional connectivity of best PCALG model. Unidirectional (bidirectional) arrows provides specific directional (feedback) information between different areas.

\section{Differential Connectivity Analysis}

The SEM parameters (path coefficients and residual variances) of the best PCALG functional network on the aforementioned brain regions and connections were fitted in a multigroup SEM analysis. This model proved to be significantly different between pairwise group comparisons: $G R N+$ vs. $G R N-\left(\mathrm{X} 2 \mathrm{diff}=35.2_{21}\right.$, $P=0.027), G R N+$ vs. HC (X2diff $\left.=38.6_{21}, P=0.011\right)$, and $G R N-$ vs. HC (X2diff $=53.8_{21}, P<0.001$ ). The significant differential links among the brain regions found in $G R N+$ and $G R N-$ are shown in Figure 3. In contrast to GRN-, FTLD GRN+ showed a different complex pattern of effective connectivity in parietotemporal cortex, bilaterally. A reduced covariance between the Pariet and Tpol and an increased connectivity between the posterior Temp and TPol and between the posterior Temp and Pariet were evident (Fig. 3A). On the other hand, GRN- presented a reduced connectivity between the Orbit and AntCing and between the Temp and DLPFC (on the right), whereas an increased covariance between the Orbit and TPol, bilaterally, was found (Fig. 3B). Finally, both GRN- and GRN+ showed a reduced connectivity between the DLPFC and Orbit.

\section{DISCUSSION}

The predominant pathologic involvement of frontal and temporal regions is the signature of FTLD, and this involvement is a supportive criterion for the clinical diagnosis of the disease. However, in the last decade, the growth of neuroimaging techniques and the application of complex statistical methods have highlighted that beyond considering anatomic regions the 




FIGURE 3. Differential functional connectivity in FTLD patients with or without Granulin mutations. Red solid lines indicate significant enhanced pathways, and broken lines indicate significant reduced pathways $(P<0.05)$. (A) FTLD due to Granulin mutation. (B) Frontotemporal dementia without known pathogenic mutations.

involvement of wide neural networks—where single regions were functionally correlated-are of interest $(10,25)$. This correlation has allowed a more precise definition of the neuroanatomic correlates of the pathologic hallmarks, giving significant clues to the molecular underpinnings of the disease itself (26-28).

Furthermore, the study of cases with disease-causing mutations within GRN, MAPT, and, more recently, the expanded hesanucleotide repeat insertion within C9orf72 showed overlap and high variability of these different neuropathologic processes $(5,21,29)$. How the alteration of a specific causative gene (i.e., $G R N$ ) leads to the involvement of specific brain regions and the impairment of several brain networks is still unclear $(30,31)$.

In the present study, we evaluated the effective connectivity in autosomal-dominant $G R N$-related FTLD, as compared with FTLD patients without pathogenetic GRN mutations and HCs, applying a statistical approach (path analysis) based on SEM on SPECT images. Considering previous data on a founder effect (32), we selected only apparently unrelated FTLD GRN+ patients to reduce the potential biases. The direct whole-brain comparison showed greater damage in the DLPFC in FTLD GRN+ than FTLD GRN-, as shown in Figure 2 and Table 2. Thus, the theoretic framework of the pathway analysis allowed us to overcome the whole-brain alterations of the perfusion pattern and to focus on large-scale network involvement.

In the present work, we reported that the signature of GRN disease was a significant disconnection between the Pariet and TPol, with a concomitant increased connectivity from the posterior Temp to the TPol and the parietal lobe. Furthermore, in the GRN- group, a disconnection between DLPFC and Temp was reported, with a relative hyperconnectivity from the Orbit and the TPol, bilaterally.

Our results are in line with previous literature on $G R N$-related FTLD assessed by structural imaging. It has been demonstrated that patients carrying $G R N$ mutations had greater frontoparietal gray matter atrophy and involvement of long intrahemispheric association white matter tracts $(11,22)$. Furthermore, data on functional brain networks underlined the selective and specific involvement of parietal networks in autosomal-dominant GRN disease $(9,33,34)$. Interestingly, connectivity analysis showed a bilateral temporoparietal disconnection. This finding is probably related to the different (left or right) parietal hypoperfusion in the $G R N+$ group and is in line with literature on $G R N$-related FTLD $(8,35)$. On the other hand, other FTLDs showed greater frontotemporal damage than $G R N$ mutations.

In line with this evidence, our work confirms and extends previous studies suggesting that different proteinopathies lead to FTLD by a selective vulnerability of brain networks (36) and likely begin from distinct brain regions that act as a hublike epicenter (37).

As interestingly suggested by Warren et al. (38), neurodegenerative dementias, as well as FTLD with different neuropathologic substrate, might result from the progressive spreading of the neurodegenerative process from a defined epicenter in the target network over common transneural pathways. This concept, defined as molecular nexopathies, hypothesized that GRN mutations cause a specific and preferential involvement of long intrahemispheric tracts between target networks and off-target pathways (i.e., temporoparietal regions). 
More research is needed to bridge the gap between molecular aspects of FTLD and the neuropathologic alterations driving the clinical and radiologic presentation $(39,40)$. Large international initiatives with multimodal imaging techniques with high-level statistical tools are required to confirm and extend the field of the molecular nexopathies.

\section{CONCLUSION}

Our data showed a selective functional disconnection of the temporoparietal network in patients with $G R N+$ and, in addition, suggested a potential complex compensative mechanism involving temporotemporal connections. Moreover, the present study argued that compensative networks might be identified in FTLD without $G R N$ mutations, representing potential targets for nonpharmacological approaches.

\section{DISCLOSURE}

The costs of publication of this article were defrayed in part by the payment of page charges. Therefore, and solely to indicate this fact, this article is hereby marked "advertisement" in accordance with 18 USC section 1734 . No potential conflict of interest relevant to this article was reported.

\section{REFERENCES}

1. Ratnavalli E, Brayne C, Dawson K, Hodges JR. The prevalence of frontotemporal dementia. Neurology. 2002;58:1615-1621.

2. Borroni B, Alberici A, Grassi M, et al. Is frontotemporal lobar degeneration a rare disorder? Evidence from a preliminary study in Brescia county, Italy. J Alzheimers Dis. 2010;19:111-116.

3. Seelaar H, Rohrer JD, Pijnenburg YA, Fox NC, van Swieten JC. Clinical, genetic and pathological heterogeneity of frontotemporal dementia: a review. J Neurol Neurosurg Psychiatry. 2011;82:476-486.

4. Rohrer JD, Warren JD. Phenotypic signatures of genetic frontotemporal dementia. Curr Opin Neurol. 2011;24:542-549.

5. Whitwell JL, Jack CR Jr, Baker M, et al. Voxel-based morphometry in frontotemporal lobar degeneration with ubiquitin-positive inclusions with and without progranulin mutations. Arch Neurol. 2007;64:371-376.

6. Whitwell JL, Przybelski SA, Weigand SD, et al. Distinct anatomical subtypes of the behavioural variant of frontotemporal dementia: a cluster analysis study. Brain. 2009;132:2932-2946.

7. Rohrer JD, Ridgway GR, Modat M, et al. Distinct profiles of brain atrophy in frontotemporal lobar degeneration caused by progranulin and tau mutations. Neuroimage. 2010;53:1070-1076.

8. Beck J, Rohrer JD, Campbell T, et al. A distinct clinical, neuropsychological and radiological phenotype is associated with progranulin gene mutations in a large UK series. Brain. 2008;131:706-720.

9. Seeley WW, Crawford R, Rascovsky K, et al. Frontal paralimbic network atrophy in very mild behavioral variant frontotemporal dementia. Arch Neurol. 2008;65:249-255.

10. Borroni B, Alberici A, Cercignani M, et al. Granulin mutation drives brain damage and reorganization from preclinical to symptomatic FTLD. Neurobiol Aging. 2012;33:2506-2520.

11. Rohrer JD, Geser F, Zhou J, et al. TDP-43 subtypes are associated with distinct atrophy patterns in frontotemporal dementia. Neurology. 2010;75:2204-2211.

12. McIntosh AR, Gonzalez-Lima F. Structural equation modeling and its application to network analysis in functional brain imaging. Hum Brain Mapp. 1994; $1-2: 2-22$.
13. Rascovsky K, Hodges JR, Knopman D, et al. Sensitivity of revised diagnostic criteria for the behavioural variant of frontotemporal dementia. Brain. 2011; 134:2456-2477.

14. Gorno-Tempini ML, Hillis AE, Weintraub S, et al. Classification of primary progressive aphasia and its variants. Neurology. 2011;76:1006-1014.

15. American Psychiatric Association (APA). Diagnostic and Statistical Manual of Mental Disorders: DSM-IV-TR. Arlington, VA: APA; 2000.

16. Dickson DW, Kouri N, Murray ME, Josephs KA. Neuropathology of frontotemporal lobar degeneration-tau (FTLD-tau). J Mol Neurosci. 2011;45:384-389.

17. Mackenzie IR. The neuropathology and clinical phenotype of FTD with progranulin mutations. Acta Neuropathol. 2007;114:49-54.

18. Whitwell JL, Josephs KA. Neuroimaging in frontotemporal lobar degenerationpredicting molecular pathology. Nat Rev Neurol. 2011;8:131-142.

19. Maldjian JA, Laurienti PJ, Kraft RA, Burdette JH. An automated method for neuroanatomic and cytoarchitectonic atlas-based interrogation of fMRI data sets. Neuroimage. 2003;19:1233-1239.

20. Cilia R, Cho SS, van Eimeren T, et al. Pathological gambling in patients with Parkinson's disease is associated with fronto-striatal disconnection: a path modeling analysis. Mov Disord. 2011;26:225-233.

21. Whitwell JL, Weigand SD, Boeve BF, et al. Neuroimaging signatures of frontotemporal dementia genetics: C9ORF72, tau, progranulin and sporadics. Brain. 2012;135:794-806.

22. Whitwell JL, Avula R, Senjem ML, et al. Gray and white matter water diffusion in the syndromic variants of frontotemporal dementia. Neurology. 2010;74:12791287.

23. Spirtes P, Glymour CN, Scheines R. Causation, Prediction, and Search. Cambridge, MA: The MIT Press. 2000.

24. Kline R. Principles and Practice of Structural Equation Modeling. New York, NY: The Guilford Press; 2010.

25. Zhou J, Greicius MD, Gennatas ED, et al. Divergent network connectivity changes in behavioural variant frontotemporal dementia and Alzheimer's disease. Brain. 2010;133:1352-1367.

26. Seeley WW, Crawford RK, Zhou J, Miller BL, Greicius MD. Neurodegenerative diseases target large-scale human brain networks. Neuron. 2009;62:42-52.

27. Pievani M, de HW, Wu T, Seeley WW, Frisoni GB. Functional network disruption in the degenerative dementias. Lancet Neurol. 2011;10:829-843.

28. Irish M, Piguet O, Hodges JR. Self-projection and the default network in frontotemporal dementia. Nat Rev Neurol. 2011;8:152-161.

29. Rohrer JD, Lashley T, Schott JM, et al. Clinical and neuroanatomical signatures of tissue pathology in frontotemporal lobar degeneration. Brain. 2011;134: 2565-2581.

30. Ward ME, Miller BL. Potential mechanisms of progranulin-deficient FTLD. J Mol Neurosci. 2011;45:574-582.

31. Sun L, Eriksen JL. Recent insights into the involvement of progranulin in frontotemporal dementia. Curr Neuropharmacol. 2011;9:632-642.

32. Borroni B, Bonvicini C, Galimberti D, et al. Founder effect and estimation of the age of the progranulin Thr272fs mutation in 14 Italian pedigrees with frontotemporal lobar degeneration. Neurobiol Aging. 2011;32:555-558.

33. Seeley WW. Selective functional, regional, and neuronal vulnerability in frontotemporal dementia. Curr Opin Neurol. 2008;21:701-707.

34. Borroni B, Alberici A, Premi E, et al. Brain magnetic resonance imaging structural changes in a pedigree of asymptomatic progranulin mutation carriers. Rejuvenation Res. 2008;11:585-595.

35. Whitwell JL, Xu J, Mandrekar J, et al. Frontal asymmetry in behavioral variant frontotemporal dementia: clinicoimaging and pathogenetic correlates. Neurobiol Aging. 2013;34:636-639.

36. Raj A, Kuceyeski A, Weiner M. A network diffusion model of disease progression in dementia. Neuron. 2012;73:1204-1215.

37. Zhou J, Gennatas ED, Kramer JH, Miller BL, Seeley WW. Predicting regional neurodegeneration from the healthy brain functional connectome. Neuron. 2012;73:1216-1227.

38. Warren JD, Rohrer JD, Hardy J. Disintegrating brain networks: from syndromes to molecular nexopathies. Neuron. 2012;73:1060-1062.

39. Davion S, Johnson N, Weintraub S, et al. Clinicopathologic correlation in PGRN mutations. Neurology. 2007;69:1113-1121.

40. McDade E, Boeve BF, Burrus TM, et al. Similar clinical and neuroimaging features in monozygotic twin pair with mutation in progranulin. Neurology. 2012;78:1245-1249. 